\title{
The Effect of Reciprocal-Space Sampling and Basis Set Quality on the Calculated Conductance of a Molecular Junction
}

\author{
R. C. HOFT, M. J. FORD*, and M. B. CORTIE \\ Institute for Nanoscale Technology, University of Technology Sydney, PO Box 123, \\ Broadway NSW 2007, Australia
}

(Received)

\begin{abstract}
We perform density functional theory and non-equilibrium Green's Function calculations of the conductance of a gold wire and a 1,4-phenylenedimethanethiol (XYL) molecule adsorbed between $\mathrm{Au}(111)$ electrodes using the TranSIESTA software package. The effect of varying different computational parameters is investigated. We find that the conductance is more sensitive to the reciprocal space sampling grid than the quality of the basis set employed. The conductance can vary up to a factor of five as a result of the choice of computational parameters. We report a set of computational parameters that yields a well-converged conductance value.
\end{abstract}

PACS numbers: 73.63.-b, 73.40.Gk, 85.35.-p

\section{Introduction}

Molecular electronics is an active area of research with the eventual goal of building an electronic device with molecules as its active components [1]. Recent Corresnondino author email: mike ford $a$ uts edu au 
advances in both the experimental [2] and theoretical [3] realm concerning the conductance of individual molecules are bringing this goal closer to reality. However a cause for concern is the large discrepancies that still exist between available data, both experimental and theoretical [4].

Molecules with thiol endgroups form strong bonds to $\mathrm{Au}(111)$ substrates and their formation of self-assembled monolayers (SAMs) is well studied [5]. For this reason the thiol 'alligator-clip' is commonly used in molecular conduction experiments. Many experiments probe the current-voltage response of a SAM using scanning tunneling spectroscopy (STS). Here the current between an STM tip and substrate under an applied bias [6-14] at fixed tip height is measured. Among other experimental tools are mechanical break junctions [15-17] and atomic force microscopes (AFM) with a conducting tip [18]. Various authors have claimed to measure the $i(V)$ response of an individual molecule, e.g. [13, 14, 16, 18].

Theoretically, experimental setups are usually approximated by two semi-infinite $\mathrm{Au}(111)$ slabs sandwiching the molecule under study. The theoretical determination of the current passing between these electrodes is complicated beyond the usual electronic structure calculation by the fact that the system, under an applied bias between the electrodes, is not in equilibrium. Density functional theory is typically used as the starting point for conductance calculations, and yields the equilibrium electronic structure of the molecule plus electrodes. The transmission function and hence current is then calculated according to the non-equilibrium Green's functions method (NEGF). This combination is now an established tool for ab-initio molecular transport calculations (see for example a recent review by Lindsay and Ratner [19]). The choice of basis functions and sampling of 
the Brillouin zone are critical factors that can affect the result when calculating the electronic structure. Care has to be taken that the result is converged with respect to these parameters, at least to the desired level of accuracy. Many molecular conduction results using the above technique now appear in the literature, but no thorough investigation has been published to clarify the effect of these parameters. Here we address this issue by studying two prototypical systems: an infinite one-dimensional gold chain and $\mathrm{Au}(111)-$ XYL-Au(111) where XYL, or 1,4-phenylenedimethanethiol, is a short, thiol-terminated aromatic molecule that easily self-assembles on a gold substrate $[9,20,21]$.

\section{Method}

The gold chain and surface adsorption geometries were initially optimized with the SIESTA software package [22], a density functional code employing a linear combination of numerical atomic orbitals as basis functions. The basis set consists of double zeta plus single polarization orbital for each atom. The orbitals have finite extent in space, the cutoff radius being defined by an "energy cutoff" parameter which specifies the excitation energy due to this confinement. This parameter is set to $5 \mathrm{mRy}$, which corresponds to a cut-off radius for the gold atoms of $3.8 \AA$ for s-orbitals and $2.7 \AA$ for dorbitals. The local density approximation (LDA) to the exchange-correlation functional is used, incorporating the self-interaction correction by Perdew and Zunger [23]. Calculations were spin-polarised with the (111) surface represented by a 3x3 atom unit cell, 4 layers thick. The surface layer is not relaxed during optimization. A reciprocal space grid with $5 \times 5 k$-points in the plane parallel to the surface is constructed according 
to the method of Monkhorst and Pack [24]. We have previously examined the reliability of this type of calculation against the computational conditions used [25]; the parameters used here correspond to intermediate level I in this previous work and yield adsorption energies that are reliable to better than $0.45 \mathrm{eV}$. Higher level calculations improve the calculated adsorption energy but do not change adsorption geometries. Here we are concerned only at locating the minimum adsorption geometry rather than calculating a chemically accurate binding energy. Note that the optimum geometry is determined for the XYL molecule adsorbed through one of the thiol groups to a single gold surface. It is then assumed in the transport calculation, where the molecule spans two surfaces, or electrodes, that the same optimized configuration occurs at both electrodes.

The relaxed geometry is then input into the transport calculation using the ATK package, based on the original TransSIESTA [26]. This uses the SIESTA method to obtain the electronic structure and calculates transport by using the non-equilibrium Green's function method. This code has now been used extensively in the literature, for example, other studies have been undertaken to investigate the effect of different bonding geometries of small organic molecules between gold electrodes on the current-voltage characteristics of the system [27-29].

[Insert figure 1 about here]

The molecule with two layers of Au atoms on either side is modeled as the device region, connected on the left and right by semi-infinite electrodes (Fig. 1b). Including two layers in the central region is enough to ensure that the bulk approximation is accurate for the electrodes. A test case calculation with three layers of gold included in the central region 
yielded no change in the result. In the Au chain calculations, three atoms are included in the device region (Fig. 1a).

We again use the local density approximation with parametrisation by Perdew and Zunger. In the interest of time the calculations are done without spin-polarization. This does not introduce an error, since the above (spin polarized) SIESTA optimisation yielded optimised geometries with a total spin of zero, as expected. The effects of the basis set size describing the gold atoms, the orbital energy cutoff and the number of $k$-points used in the plane parallel to the surface are investigated within the ATK package. The basis sets used on the atoms within the molecule spanning the electrodes are kept fixed at double zeta plus polarization orbitals (DZP) for each atom. The calculation time is much more sensitive to the basis set size on the gold atoms; this is alternated between DZP $\left(\zeta_{\mathrm{Au}}\right.$ $=2$ ) and single zeta plus polarization $\left(\zeta_{\mathrm{Au}}=1\right)$. The orbital confinement parameter $E_{c}$ is varied between $0.02 \mathrm{mRy}$ and $20 \mathrm{mRy}$. In the case of transport calculations the determination of the transmission function is a post-electronic structure calculation. The self-consistent electronic structure is characterized by the density matrix, given within the NEGF formalism by [30]

$$
\rho=\frac{1}{2 \pi} \int_{-\infty}^{\infty} \mathrm{d} E \cdot\left[f\left(E-\mu_{1}\right) G_{\mathrm{d}} \Gamma_{1} G_{\mathrm{d}}^{*}+f\left(E-\mu_{2}\right) G_{\mathrm{d}} \Gamma_{2} G_{\mathrm{d}}^{*}\right]
$$

where $f\left(E-\mu_{1(2)}\right)$ and $\Gamma_{1(2)}$ are the Fermi functions and coupling matrices of the left (right) electrodes and $\mathrm{G}_{\mathrm{d}}$ is the device Green's function in the presence of the electrodes.

The electron density and resulting Kohn-Sham operator can be used to evaluate the Hamiltonian at a denser $k$-point grid, using [22] 


$$
H_{\mu \nu}(\vec{k})=\sum_{\mu^{\prime} \equiv \mu} \sum_{v^{\prime} \equiv v} e^{i \vec{k} \cdot\left(\vec{R}_{v}-\vec{R}_{\mu}\right)}\left\langle\phi_{\mu^{\prime}}(\vec{r})\left|\hat{f}^{\mathrm{KS}}\right| \phi_{v^{\prime}}(\vec{r})\right\rangle
$$

where $\mu^{\prime} \equiv \mu$ indicates all the periodic images of the basis function labeled $\mu$, centered at $\mathrm{R}_{n}$, without repeating the SCF at this denser grid. If the electronic structure is converged with respect to the $k$-point grid used in the SCF cycle, then the lack of selfconsistency at the denser grid should not have a large effect. From the Hamiltonian, the Green's function and hence transmission function and current is calculated from [30]

$$
I(V)=\frac{2 e}{h} \int_{-\infty}^{\infty} \mathrm{d} E \cdot\left[\operatorname{Tr}\left(\Gamma_{1} G_{\mathrm{d}} \Gamma_{2} G_{\mathrm{d}}^{*}\right)\left(f\left(E-\mu_{1}\right)-f\left(E-\mu_{2}\right)\right)\right]
$$

where the transmission function can be identified as $T(E)=\operatorname{Tr}\left(\Gamma_{1} \mathrm{G}_{\mathrm{d}} \Gamma_{2} \mathrm{G}_{\mathrm{d}}{ }^{*}\right)$.

For the calculation of the Au-XYL-Au system, we use the same number of grid points $N$ in both the $x$ and $y$ direction. We vary the number of grid points for the selfconsistent electronic structure calculation $N_{E}$ and the transmission function calculation $N_{T}$. For the gold chain, only the gamma point is required in either the SCF or transmission function calculation, as there is no periodicity in the transverse direction.

The device region is not periodic and hence no $k$-point sampling in the direction perpendicular to transport is done in the two-probe calculation. However for the calculation of the electrodes a large number of $k$-points, generally 100 , is used in this direction to ensure the metallic behaviour of the electrodes.

\section{Results And Discussion}

\subsection{Gold chain}


Fig. 2 shows the current-voltage and conductance-voltage characteristics of a gold chain, calculated with a SZP basis set $\left(\zeta_{\mathrm{Au}}=1\right)$ and $E_{c}=5$ or $10 \mathrm{mRy}$. The $I(V)$ characteristics seem virtually unaffected, but changing the energy shift parameter clearly has a larger effect on the conductance. The latter however remains close to the conductance quantum, $G_{0}=2 \mathrm{e}^{2} / \mathrm{h}=77.48 \mu \mathrm{S}$, as expected for a one-dimensional gold chain [30]. The response of the chain is almost Ohmic, but the conductance decreases slightly at higher voltages. This behaviour has been observed in similar calculations and in experiments [26]. In what follows we shall refer to the conductance in units of $G_{0}$.

[Insert figure 2 about here]

The conductance can either be calculated from the difference between successive current evaluations

$$
G_{\Delta}(V)=\frac{I(V+\Delta V)-I(V-\Delta V)}{2 \Delta V}
$$

or by the analytical derivative of the expression for the current, equation (3), which

$$
\begin{aligned}
G(V) & =\frac{2 e^{2}}{h} \int_{-\infty}^{\infty} \mathrm{d} E \cdot T(E) \frac{1}{2 k T}\left(\frac{t_{1}}{\left(1+t_{1}\right)^{2}}+\frac{t_{2}}{\left(1+t_{2}\right)^{2}}\right) \\
\text { gives } \quad t_{1} & =\exp \left(\frac{E+e V / 2}{k T}\right) \\
t_{2} & =\exp \left(\frac{E-e V / 2}{k T}\right)
\end{aligned}
$$

conductance given in Fig. $2 \mathrm{~b}$ is based on equation (4) with $\Delta V=0.2 \mathrm{~V}$ and $T=300 \mathrm{~K}$.

Although equation (5) is exact, it is not necessarily the better option, since discontinuities in the transmission function at energies close to the chemical potentials of 
the electrodes, can lead to an unreliable conductance value. This is illustrated in Fig. 3. At $0 \mathrm{~V}$ the transmission function is discontinuous near $E=0$, where the number of transmission channels changes between one and three. The zero-volt conductance evaluated from equation (5) with $T=0 \mathrm{~K}$ can therefore be 1 or 3 , depending on the exact location of the discontinuity, $\mathrm{E}=\varepsilon$. Introducing a finite temperature into equation (5) reduces the sensitivity to $\varepsilon$. The sensitivity is further reduced by using equation (4), where the evaluation of the current integrals occurs over an interval spanning $\mathrm{E}=\varepsilon$. [insert figure 3 about here]Fig. 4 shows the zero-volt conductance of the gold chain calculated from equations (4) and (5) with both $T=0$ and $300 \mathrm{~K}$. An SZP basis set is used and $E_{c}$ is varied over a wide range. Some results for a DZP basis set are also shown for comparison. 
[insert figure 4 about here]

The conductance is very sensitive to the energy shift parameter and approaches the expected value of $G=G_{0}=2 \mathrm{e}^{2} / \mathrm{h}$ as the basis set orbitals become less confined. When equation (4) is used (fig $4 \mathrm{a}$ ), the conductance is converged at $\mathrm{E}_{\mathrm{c}}=1 \mathrm{mRy}$. Convergence with respect to the orbital confinement is faster at $T=0 \mathrm{~K}$. Using equation (5) (fig 4b), the conductance is converged at $E_{\mathrm{c}}=1 \mathrm{mRy}$ when $T=0 \mathrm{~K}$, but only at $E_{\mathrm{c}}=0.1 \mathrm{mRy}$ when $T=300 \mathrm{~K}$. However, at zero temperature the convergence is discontinuous and the conductance jumps from $\mathrm{G}_{0}$ to $3 \mathrm{G}_{0}$ between $\mathrm{E}_{\mathrm{c}}=1 \mathrm{mRy}$ and $\mathrm{E}_{\mathrm{c}}=5 \mathrm{mRy}$.

At $E_{\mathrm{c}}=1 \mathrm{mRy}$ and $10 \mathrm{mRy}$ the conductance was recalculated with a DZP basis set (hollow data points). These conductance values are invariably very close to their SZP counterparts, the values when $\mathrm{E}_{\mathrm{c}}=1 \mathrm{mRy}$ and $T=0 \mathrm{~K}$ being identical.

For accurate conductance results, it is therefore imperative to use an orbital confinement corresponding to an energy shift parameter of $1 \mathrm{mRy}$ or lower. Table 1 lists the orbital confinement radii corresponding to different values of $E_{\mathrm{c}}$. Use of a DZP basis set does not provide a significant improvement over SZP and is therefore not recommended, as it implies a significant increase in computational time. For Au, an SZP basis set includes 9 orbitals per atom and a DZP basis set 15 orbitals per atom. When using a sufficiently accurate orbital confinement, the zero-temperature conductance is more accurate than the finite-temperature conductance; however, case must be taken when using a less accurate orbital confinement, since a discontinuous transmission function may lead to an incorrect conductance value. 


\section{$3.2 A u-X Y L-A u$}

[insert figure 5 about here]

The potential energy surface (PES) of XYL adsorbed on the Au(111) surface is shown in Fig. 5. The optimal binding site is slightly offset from the bridge site, towards the fcc site. The binding height is $2.0 \AA$. The minimum energy position was initially the result of a conjugate gradient (CG) optimisation of the molecule on the surface and the PES was produced by manually varying the binding site and height of the sulphur atom on the surface. This manual scanning of the molecule confirms that the CG result is a global minimum with respect to the sulphur position above the surface.

Fig. 6 shows the variation in interaction energy with two independent rotation angles of the molecule on the surface. Firstly the plane of the phenyl ring was rotated with respect to the surface to make an angle of $\theta$ degrees with the surface normal (Fig. 6a). Secondly the molecule was rotated within the plane of the phenyl ring so that an angle of $\sigma$ degrees is made between the surface normal and the vector connecting the carbon atoms in the 1 and 4 positions in the ring (Fig. 6b). The inserts in Figs. 6a and 6b clarify these rotations. In both cases the rotations are done about the sulphur atom bound to the surface. In the CG optimised geometry the rotation angles are $\theta=-21^{\circ}$ and $\sigma=0^{\circ}$. $\theta$ and $\sigma$ are then varied independently with the other angle fixed at the CG optimised value. Manually varying these rotation angles confirms that the CG result is a global minimum with respect to rotation of the molecule on the surface. The minima in Figs. 6a and $6 \mathrm{~b}$ therefore correspond to the same minimum energy structure, shown in Fig. 6c. 
The interaction energy and optimum geometry is similar to that calculated previously for other thiol-bound molecules; however, the rotation angle may be optimised more reliably by using a Z-matrix optimiser [31].

[insert figure 6 about here]

The ATK electronic structure and transport calculations are set up accordingly with the molecule bonded identically to both electrodes.

Firstly, the total energy and the eigenenergies of the highest occupied molecular orbital (HOMO) and lowest unoccupied molecular orbital (LUMO) are calculated with $E_{\mathrm{c}}$ $=20 \mathrm{mRy}$ and $\zeta_{\mathrm{Au}}=1$ (SZP), varying $N_{\mathrm{E}}$ between 1 and 13. The results are shown in Fig. 7. At $N_{\mathrm{E}}=3$ the total energy has converged to within $1 \mathrm{eV}$ (or $0.01 \mathrm{eV} /$ atom) and the HOMO and LUMO eigenenergies to within $0.005 \mathrm{eV}$ of the limiting values. Here we have assumed that using a value of $N_{e}=13$ gives a converged result.

[insert figure 7 about here]

It is not surprising that the electronic structure is sensitive to $k$-point sampling for this device configuration, which is periodic in two dimensions and therefore possesses dispersive energy bands in $k$-space. However, the total energy and HOMO and LUMO eigenenergies are seen to be well converged at the relatively modest $3 \times 3 k$-space sampling grid. It should also be noted that more reliable total energy results are obtained by including the gamma point in the $k$-space integration, which in the present code is achieved by using an odd numbered $k$-grid.

For efficiency only the $0 \mathrm{~V}$ transmission function was calculated for the XYL system. The zero-volt conductance in what follows was therefore calculated from equation (5), which does not make use of the current at finite voltages. The use of a finite 
temperature avoids the problem of a discontinuous transmission spectrum described in section 3.1.

Fig. 8 shows the zero-volt conductance of the system calculated from equation (5), using $T=300 \mathrm{~K}$, as a function of $N_{T}$ for various choices of $N_{E}, \zeta_{\mathrm{Au}}$ and $E_{c}$. Keeping $E_{\mathrm{c}}=$ $20 \mathrm{mRy}, \zeta_{\mathrm{Au}}=1$ fixed and varying $N_{\mathrm{E}}, N_{\mathrm{T}}\left(\right.$ Fig. 8a), the converged value at $N_{\mathrm{E}}=N_{\mathrm{T}}=13$ is $G=0.0401 G_{0}$. A modest $2 \times 2 k$-point grid for the SCF calculation seems sufficient for a well converged conductance value and with $N_{\mathrm{E}}=2, N_{\mathrm{T}}=5$, the zero-volt conductance is converged to within $10 \%$ of the limiting value. We use $N_{\mathrm{T}}=13$, since the calculation of the transmission function occurs post-SCF, and therefore $N_{\mathrm{T}}$ contributes only a small amount to the total computational time. This is quantified in Fig. 9. The transmission function calculation time is about two orders of magnitude smaller than the electronic structure calculation time and hence the size of the $N_{\mathrm{T}} \mathrm{x} N_{\mathrm{T}}$ grid has negligible effect on the total computation time.

[insert figure 8 about here]

In Fig. $8 \mathrm{~b}, E_{\mathrm{c}}, \zeta_{\mathrm{Au}}$ and $N_{\mathrm{E}}$ are varied simultaneously. For $N_{T}=1, G$ changes by up to a factor of three depending on the other parameters, although the errors introduced by $\zeta_{\mathrm{Au}}$ and $E_{c}$ appear to cancel. However when convergence is reached with respect to $N_{T}$, it seems that the basis set parameters (orbital confinement and basis set size) do not affect the converged value, whereas the $k$-point sampling used for the electronic structure does have an effect.

[insert figure 9 about here]

Finally we test the convergence of the total energy and zero-volt conductance with respect to the energy shift parameter, Fig. 10. We set $\zeta_{\mathrm{Au}}=1$ and $N_{\mathrm{E}}=1$. We use the 
gamma point only here in the interest of speed; although the absolute values are therefore not necessarily correct, the convergence behaviour should be unaffected. Taking the values at $\mathrm{E}_{\mathrm{c}}=0.01 \mathrm{mRy}$ to be converged, the total energy at $E_{\mathrm{c}}=2 \mathrm{mRy}$ is converged to within $4 \mathrm{eV}$ (or $0.04 \mathrm{eV} /$ atom) of the limiting value. $G$ evaluated with $N_{\mathrm{T}}=13$ is converged to within $6 \%$ at $E_{\mathrm{c}}=5 \mathrm{mRy}$. However $G$ evaluated with $N_{\mathrm{T}}=1$, is only well converged at $E_{\mathrm{c}}=0.5 \mathrm{mRy}$, to within $10 \%$ of the limiting value. This is consistent with the finding from Fig. $8 \mathrm{~b}$, i.e. when convergence is reached with respect to $N_{\mathrm{T}}$, the basis set parameters have less effect on the converged $G$ value.

[insert figure 10 about here]

The best value for the zero-volt conductance of the $\mathrm{Au}(111)-\mathrm{XYL}-\mathrm{Au}(111)$ system presented here is $G=0.0493 G_{0}$, with $\mathrm{N}_{\mathrm{E}}=3, \mathrm{~N}_{\mathrm{T}}=7, \mathrm{E}_{\mathrm{c}}=5 \mathrm{mRy}$ and $\xi_{\mathrm{Au}}=2$. This is a factor 82 larger than the experimental measurement of $G=0.0006 G_{0}[13]$. Discrepancies of this order between calculated and experimental conductances of thiol-bound molecules on gold have been noted before in DFT calculations [32, 33].

There is an additional constraint associated with the energy-shift parameter which must be considered in calculations where a vacuum gap is incorporated into the geometry. This would be the case for simulating an STM experiment, or for modeling the effect of moving the molecule away from one electrode [34]. Because the orbitals fall strictly to zero, a sufficiently large orbital confinement radius must be used in order to ensure the gap is spanned by the molecule / electrode orbitals. In other words, a small energy-shift parameter must be used in the transport calculations; the exact value of this parameter will depend upon the geometry. Table 1 gives energy-shifts and the corresponding confined radius for carbon and gold atoms; confinement will vary for 
different elements even with a common energy-shift. The confinement radii suggest that it may be desirable to use an energy-shift parameter as small as $E_{\mathrm{c}}=0.1 \mathrm{mRy}$ when there is a distance of 5-7 $\AA$ between molecule and electrode, to ensure significant orbital overlap. This is more accurate than is usually needed to obtain converged adsorption geometries and interaction energies of molecules on surfaces [25]. This is another point that has not been addressed in DFT calculations of transport in the literature. Orbital confinement is not relevant in plane wave codes, but the above discussion is applicable to all codes that use the linear combination of atom orbitals (LCAO) ansatz, where the atom-centred basis functions generally have finite spatial extent.

[insert table 1 about here]

\section{Conclusion}

In summary, we have conducted a large set of DFT electronic structure and transmission calculations on a gold chain as well as the model Au-XYL-Au system, which is a commonly studied system in the context of single-molecule conductance. For the gold chain, which requires no $k$-point sampling, we test the effect of altering the basis set parameters on the current and conductance. We find that the $I(V)$ characteristic is relatively insensitive to the basis set, but that the conductance can be highly sensitive to the basis set when the transmission function is discontinuous or rapidly changing. The orbital confinement radius appears to be more important than the number of distinct orbitals per angular momentum channel. This sensitivity can be reduced by applying a 
finite temperature or by using successive current integration to evaluate the conductance, rather than using the analytical value.

For $k$-point the Au-XYL-Au system, we test the variation of the calculated conductance of the system at equilibrium (i.e. 0 volt bias) when certain important variables are changed and find a set of parameters that appear to yield a converged conductance. Including two layers of gold atoms on either side of the device region making up the "extended molecule" is sufficient for a converged result. Changing the basis set size and the orbital confinement and the sampling for both the electronic structure and transmission calculations independently, can change the conductance by typically a factor of three, and even up to a factor of five. We find that the $k$-point sampling has a more critical effect than the basis set (size and orbital cutoff parameters).

The $k$-point sampling for the electronic structure can be coarser than that of the transmission spectrum calculation. For the Au-XYL-Au system $3 \times 3$ and $10 \times 10$ Monkhorst-Pack grids for the two calculations respectively are sufficient. With these grids, using a single zeta plus polarization orbital for gold atoms with the orbital confinement defined by a $5 \mathrm{mRy}$ energy shift parameter gives a converged conductance. The energy-shift parameter may need to be reduced when considering geometries other than the optimal binding geometry, e.g. with a gap between the molecule and one electrode.

\section{Acknowledgements}

This work was supported by the Australian Research Council and the University of Technology, Sydney. Computing facilities were provided by the Australian Centre for 
Computing and Communications (ac3) and the Australian Partnership for Advanced Computing (APAC).

\section{References}

[1] M. A. Reed. Molecular-scale electronics. Proc. IEEE, 87, 652 (1999).

[2] D. K. James and J. M. Tour. Electrical measurements in molecular electronics. Chem. Mater., 16, 4423 (2004).

[3] Y. Q. Xue and M. A. Ratner. Theoretical principles of single-molecule electronics: A chemical and mesoscopic view. Int. J. Quantum Chem., 102, 911 (2005).

[4] A. Nitzan and M. A. Ratner. Electron transport in molecular wire junctions. Science, 300, 1384 (2003).

[5] J. C. Love, L. A. Estroff, J. K. Kriebel, R. G. Nuzzo and G. M. Whitesides. Selfassembled monolayers of thiolates on metals as a form of nanotechnology. Chem. Rev., 105, 1103 (2005).

[6] R. P. Andres, J. D. Bielefeld, J. I. Henderson, D. B. Janes, V. R. Kolagunta, C. P. Kubiak, W. J. Mahoney and R. G. Osifchin. Self-assembly of a two-dimensional superlattice of molecularly linked metal clusters. Science, 273, 1690 (1996).

[7] L. A. Bumm, J. J. Arnold, M. T. Cygan, T. D. Dunbar, T. P. Burgin, L. Jones, D. L. Allara, J. M. Tour and P. S. Weiss. Are single molecular wires conducting? Science, 271, 1705 (1996).

[8] S. Datta, W. D. Tian, S. H. Hong, R. Reifenberger, J. I. Henderson and C. P. Kubiak. Current-voltage characteristics of self-assembled monolayers by scanning tunneling microscopy. Phys. Rev. Lett., 79, 2530 (1997).

[9] M. Dorogi, J. Gomez, R. Osifchin, R. P. Andres and R. Reifenberger. RoomTemperature Coulomb-Blockade from a Self-Assembled Molecular Nanostructure. Phys. Rev. B, 52, 9071 (1995).

[10] T. Ishida, W. Mizutani, N. Choi, U. Akiba, M. Fujihira and H. Tokumoto. Structural effects on electrical conduction of conjugated molecules studied by scanning tunneling microscopy. J. Phys. Chem. B, 104, 11680 (2000).

[11] K. Moth-Poulsen, L. Patrone, N. Stuhr-Hansen, J. B. Christensen, J. P. Bourgoin and T. Bjornholm. Probing the effects of conjugation path on the electronic transmission through single molecules using scanning tunneling microscopy. Nano Lett., 5, 783 (2005).

[12] J. J. W. M. Rosink, M. A. Blauw, L. J. Geerligs, E. van der Drift and S. Radelaar. Tunneling spectroscopy study and modeling of electron transport in small conjugated azomethine molecules. Phys. Rev. B, 62, 10459 (2000).

[13] X. Y. Xiao, B. Q. Xu and N. J. Tao. Measurement of single molecule conductance: Benzenedithiol and benzenedimethanethiol. Nano Lett., 4, 267 (2004).

[14] B. Q. Xu and N. J. J. Tao. Measurement of single-molecule resistance by repeated formation of molecular junctions. Science, 301, 1221 (2003). 
[15] T. Bohler, J. Grebing, A. Mayer-Gindner, H. V. Lohneysen and E. Scheer. Mechanically controllable break-junctions for use as electrodes for molecular electronics. Nanotechnology, 15, S465 (2004).

[16] M. A. Reed, C. Zhou, C. J. Muller, T. P. Burgin and J. M. Tour. Conductance of a molecular junction. Science, 278, 252 (1997).

[17] J. Reichert, R. Ochs, D. Beckmann, H. B. Weber, M. Mayor and H. von Lohneysen. Driving current through single organic molecules. Phys. Rev. Lett., 88, 176804 (2002).

[18] X. D. Cui, A. Primak, X. Zarate, J. Tomfohr, O. F. Sankey, A. L. Moore, T. A. Moore, D. Gust, G. Harris and S. M. Lindsay. Reproducible measurement of singlemolecule conductivity. Science, 294, 571 (2001).

[19] S. M. Lindsay and M. A. Ratner. Molecular transport junctions: Clearing mists. Adv. Mater., 19, 23 (2007).

[20] J. I. Henderson, S. Feng, G. M. Ferrence, T. Bein and C. P. Kubiak. Self-assembled monolayers of dithiols, diisocyanides, and isocyanothiols on gold: 'Chemically sticky' surfaces for covalent attachment of metal clusters and studies of interfacial electron transfer. Inorg. Chim. Acta, 242, 115 (1996).

[21] H. M. Zareie, A. M. McDonagh, J. Edgar, M. J. Ford, M. B. Cortie and M. R. Phillips. Controlled assembly of 1,4-phenylenedimethanethiol molecular nanostructures. Chem. Mater., 18, 2376 (2006).

[22] J. M. Soler, E. Artacho, J. D. Gale, A. Garcia, J. Junquera, P. Ordejon and D. Sanchez-Portal. The SIESTA method for ab initio order-N materials simulation. J. Phys.: Condens. Matter, 14, 2745 (2002).

[23] J. P. Perdew and A. Zunger. Self-interaction correction to density-functional approximations for many-electron systems. Phys. Rev. B, 23, 5048 (1981).

[24] H. J. Monkhorst and J. D. Pack. Special points for Brillouin-zone integrations. Phys. Rev. B, 13, 5188 (1976).

[25] M. J. Ford, R. C. Hoft and A. McDonagh. First principles theoretical prediction of a new class of self-assembled monolayer: ethynylbenzenes on gold. Journal of Physical Chemistry B, 109, 20387 (2005).

[26] M. Brandbyge, J. L. Mozos, P. Ordejon, J. Taylor and K. Stokbro. Densityfunctional method for nonequilibrium electron transport. Phys. Rev. B, 65, 165401 (2002).

[27] K. H. Muller. Effect of the atomic configuration of gold electrodes on the electrical conduction of alkanedithiol molecules. Phys. Rev. B, 73, 045403 (2006).

[28] Y. B. Hu, Y. Zhu, H. J. Gao and H. Guo. Conductance of an ensemble of molecular wires: A statistical analysis. Phys. Rev. Lett., 95, 156803 (2005).

[29] H. Basch, R. Cohen and M. A. Ratner. Interface geometry and molecular junction conductance: Geometric fluctuation and stochastic switching. Nano Lett., 5, 1668 (2005).

[30] S. Datta, Quantum Transport: Atom to Transistor, Cambridge University Press, Cambridge, 2005.

[31] M. J. Ford, R. C. Hoft and J. D. Gale. Adsorption and dimerisation of thiol molecules on $\mathrm{Au}(111)$ using a Z-matrix approach in density functional theory. Mol. Sim., 32, 1219 (2006).

[32] M. Di Ventra, S. T. Pantelides and N. D. Lang. First-principles calculation of transport properties of a molecular device. Phys. Rev. Lett., 84, 979 (2000). 
[33] K. Stokbro, J. Taylor, M. Brandbyge, J. L. Mozos and P. Ordejon. Theoretical study of the nonlinear conductance of Di-thiol benzene coupled to $\mathrm{Au}(111)$ surfaces via thiol and thiolate bonds. Comp. Mater. Sci., 27, 151 (2003).

[34] R. C. Hoft, M. Ford and M. B. Cortie. Prediction of increased tunneling current by bond length stretch in molecular break junctions. Chem. Phys. Lett., 429, 503 (2006). 